Nota clínica

\title{
Estudio de la microcirculación cerebral en el traumatismo craneoencefálico mediante el sistema Side Stream Dark Field (SDF)
}

\author{
JON PÉREZ-BÁRCENAa , JAVIER IBÁÑEZ ${ }^{b}$, MARTA BRELL ${ }^{b}$, PEDRO LLINÁS ${ }^{b}$, \\ JOSEP MARIA ABADAL ${ }^{\text {a }}$ Y JUAN ANTONIO LLOMPART-POU ${ }^{a}$
}

aServicio de Medicina Intensiva. Hospital Universitario Son Dureta. Palma de Mallorca. Islas Baleares. España.
bServicio de Neurocirugía. Hospital Universitario Son Dureta. Palma de Mallorca. Islas Baleares. España.

Entre las distintas causas de la hipoxia tisular en el traumatismo craneoencefálico grave (TCEG) se encuentran las alteraciones de la microcirculación, que se pueden estudiar in vivo mediante el sistema SDF (Side Stream Dark Field). Dicho sistema se basa en un pequeño microscopio de mano que emite una luz polarizada ortogonal. La imagen obtenida del lecho capilar luego puede analizarse mediante un software específico que permite cuantificar la longitud y la densidad de los capilares sanguíneos.

Presentamos el caso de un paciente con TCEG, intervenido para evacuación de una contusión cerebral. Durante la intervención se grabó, mediante el microscopio SDF, la microcirculación cerebral en la zona lesionada. Los resultados obtenidos se compararon con el patrón de un paciente intervenido de forma electiva por un aneurisma incidental. Tanto la imagen como el análisis cuantitativo muestran diferencias en el estado de la microcirculación cerebral, y se aprecia una reducción en la longitud y la densidad de los vasos de pequeño calibre en el paciente con traumatismo.

El sistema SDF permite el estudio in vivo y de forma no invasiva de la microcirculación cerebral, y puede contribuir a profundizar en la fisiopatología de la hipoxia tisular postraumática.

PALABRAS CLAVE: Traumatismo craneoencefálico. Isquemia. Microcirculación.

Correspondencia: Dr J. Pérez Bárcena.

Servicio de Medina Intensiva. Hospital Universitario Son Dureta.

Andrea Doria, 55. 07014 Palma de Mallorca. Illes Balears. España.

Correo electrónico: jperez@hsd.es

Manuscrito aceptado el 21-4-2008.
STUDY OF A BRAIN MICROCIRCULATION IN CRANIOENCEPHALIC TRAUMA USING THE SIDE STREAM FIELD (SDF) SYSTEM

Posttraumatic tissular hypoxia can be due to multiple causes, including microcirculation disturbances, which can be studied with the SDF (Side Stream Dark Field) system. This system is based on a small hand-held microscope that eliminates directly reflected green polarised light from an organ surface using an orthogonal analyser. It offers clear images of red and white blood cells flow through microcirculation. Specific software is later used to determine the lenght and density of microvessels.

We present a case of a TBI patient who required surgical evacuation of a brain contusion. Images of the microcirculatory bed were recorded with the SDF microscope and compared with a normal pattern obtained from another patient who was operated on for an unruptured cerebral aneurysm. Both imaging and quantitative analyses showed significant differences in the cerebral microcirculatory status in these patients. Total length and density of vessels were markedly reduced in the TBI patient.

SDF imaging allows direct and non-invasive in vivo observation of cerebral microcirculation, and may allow us to deepen our knowledge of the pathophysiology of posttraumatic brain ischemia.

KEY WORDS: Traumatic brain injury. Ischemia. Microcirculation.

\section{INTRODUCCIÓN}

La importancia clínica de las lesiones secundarias en los pacientes que han sufrido un traumatismo craneoencefálico grave (TCEG) ha generado un gran 
interés por profundizar en el conocimiento de su fisiopatología ${ }^{1,2}$. Las lesiones secundarias de mayor significación en el pronóstico de los pacientes con TCEG son los fenómenos de isquemia e hipoxia cerebral postraumática y la hipertensión intracraneal ${ }^{2,3}$. La isquemia cerebral se define por un flujo sanguíneo cerebral $<18 \mathrm{ml} / 100 \mathrm{~g} / \mathrm{min}$, y se presenta aproximadamente en el $30 \%$ de los pacientes que han sufrido un $\mathrm{TCEG}^{4}$, y hasta en el $90 \%$ de los pacientes fallecidos tras un $\mathrm{TCEG}^{5,6}$. Su detección y su tratamiento han sido los principales objetivos en el manejo de los pacientes con TCEG. Para ello, se han empleado distintos métodos de diagnóstico por imagen y métodos de evaluación metabólica tisular general o regional in vivo. Esta monitorización multimodal ha demostrado que la isquemia/hipoxia tisular en los pacientes con TCEG es habitualmente multifactorial, y que un único modelo que pretenda interpretar estas alteraciones, fundamentado exclusivamente en causas relacionadas con descensos del flujo sanguíneo cerebral y alteraciones de la presión de perfusión cerebral, es claramente insuficiente ${ }^{7-10}$. Por ello se hace necesario avanzar hacia la elaboración de nuevos conceptos que nos ayuden a interpretar adecuadamente la fisiopatología de la isquemia/hipoxia tisular, que actualmente ya puede detectarse en el ámbito clínico con monitorización mediante microdiálisis y oximetría tisular. Sin embargo, y debido a limitaciones metodológicas, con frecuencia nos encontramos ante la imposibilidad de hacer un diagnóstico exacto y, frecuentemente, el diagnóstico etiológico se establece por exclusión $^{11}$.

En este sentido, en el año 2000 se publicó un estudio en que, mediante microscopia electrónica y obtención de moldes de corrosión vascular, se pudo comprobar post mortem las alteraciones morfoestructurales de la microcirculación en pacientes que habían sufrido un $\mathrm{TCEG}^{12}$. El estudio de dichas alteraciones in vivo estaba limitado por la falta de una técnica apropiada. Recientemente se ha introducido la imagen mediante el sistema SDF (Sidestream Dark Field) (MicroScan ${ }^{\circledR}$; Microvision Medical; Países Bajos) para el estudio in vivo y de forma no invasiva de la microcirculación; su empleo ha sido validado mediante la comparación con la capilaroscopia, que puede considerarse el estándar ${ }^{13}$.

Presentamos un caso clínico en el que empleamos el sistema SDF para visualizar y cuantificar el estado de la microcirculación en un paciente con TCEG y lo comparamos con un caso control.

\section{CASO CLÍNICO}

Varón de 26 años, sin antecedentes de interés, que sufre un atropello en la vía pública. Es recogido por una ambulancia medicalizada en GCS de 4 puntos (M2-O1-V1). Se procedió a su sedación, intubación y traslado a nuestro centro.

En la tomografía computarizada (TC) de ingreso se evidenciaron contusiones hemorrágicas temporales bilaterales en cápsula interna derecha y otra en cápsula interna y núcleo caudado izquierdos; edema cerebral difuso con obliteración de surcos y cisternas de la base del cráneo y pequeña colección extraaxial temporoparietal bilateral; línea media centrada, fractura de la base del cráneo y fractura temporoparietal derecha con extensión al peñasco. Se decidió monitorizar al paciente con un sensor de presión intracraneal (PIC) y otro de presión tisular de oxígeno que se colocaron en el lóbulo frontal derecho.

Las cifras iniciales de PIC fueron elevadas, pero se pudieron controlar con sedación y relajación. Sin embargo, a partir de las 48 horas del ingreso la PIC volvió a subir, por lo que se realizó una TC craneal de control (tercera desde el ingreso), que mostró un aumento de la contusión temporoparietal derecha y se calculó un volumen aproximado de $28 \mathrm{ml}$. Se decidió la intervención quirúrgica, y se efectuó una craneotomía frontotemporal derecha y evacuación de la contusión. Ante la imposibilidad de cerrar la duramadre por un considerable swelling cerebral, no se repuso el colgajo óseo.

Tras la cirugía, la PIC estuvo controlada y se pudo retirar progresivamente todas las medidas terapéuticas. El paciente evolucionó satisfactoriamente y, finalmente, fue dado de alta a planta a los 20 días con un GCS de 10 puntos (O4-M5-V1).

Durante la cirugía, se realizó la grabación de las imágenes de la microcirculación. Previamente, el comité de investigación de nuestro hospital había aprobado el estudio. Asimismo, el paciente utilizado como control y los familiares del paciente del caso clínico firmaron el consentimiento informado; en ambos casos autorizaron la realización de la técnica.

Una vez evacuada la contusión, y antes de iniciar el cierre por planos, se grabaron 8 secuencias de $10 \mathrm{~s}$. Las grabaciones se realizaron sobre la corteza cerebral, en el límite de la resección quirúrgica (área de penumbra traumática) (fig. 1).

El paciente control era un varón de 51 años, sin antecedentes patológicos de interés, intervenido quirúrgicamente de forma electiva por un aneurisma cerebral incidental de arteria cerebral media derecha. No presentaba lesiones de ningún tipo en el parénquima, por lo que lo consideramos un buen ejemplo del estado normal de la microcirculación cerebral. Las imágenes que se muestran se obtuvieron también sobre la corteza cerebral de los lóbulos frontal y temporal (fig. 2).

Al comparar ambas imágenes, se aprecia que el fondo de la figura 1 es negro debido a la gran cantidad de hematíes extravasados. Además, en la figura 1, destaca una imagen en forma de telaraña que podría corresponder a la pía-aracnoides. La mayor diferencia entre ambas imágenes es la densidad de vasos. En la figura 1 apenas se visualizan vasos, a pesar de que la imagen corresponde a la corteza cerebral pericontusional. Además, los pocos vasos que se observan son difícilmente identificables, al comparar esta imagen con la del paciente control.

Numéricamente, estas diferencias se encuentran reflejadas en la tabla 1. La longitud total de los vasos en el paciente control fue $5.120 \mu \mathrm{m}$ y en el paciente con traumatismo, de $625 \mu \mathrm{m}$. Respecto a la densidad, 


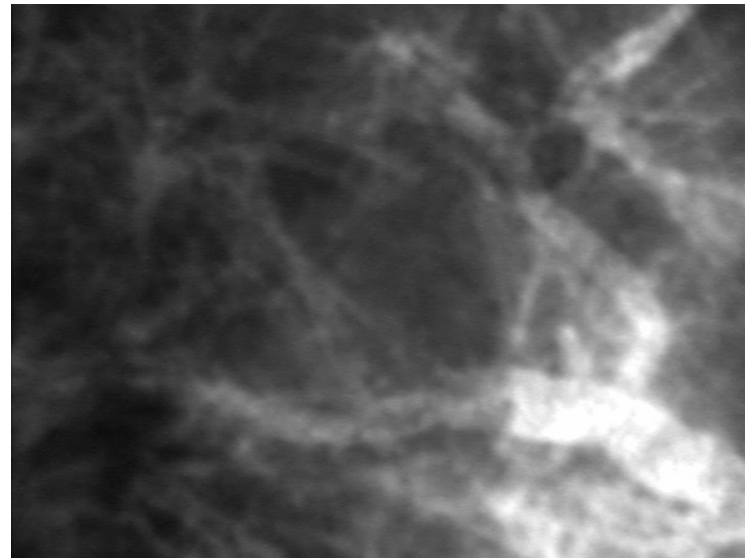

Figura 1. Imagen de la microcirculación de un paciente con traumatismo craneoencefálico grave y contusión cerebral. La grabación se realizó sobre la corteza cerebral, en el límite de la resección quirúrgica de la contusión (área de penumbra traumática).

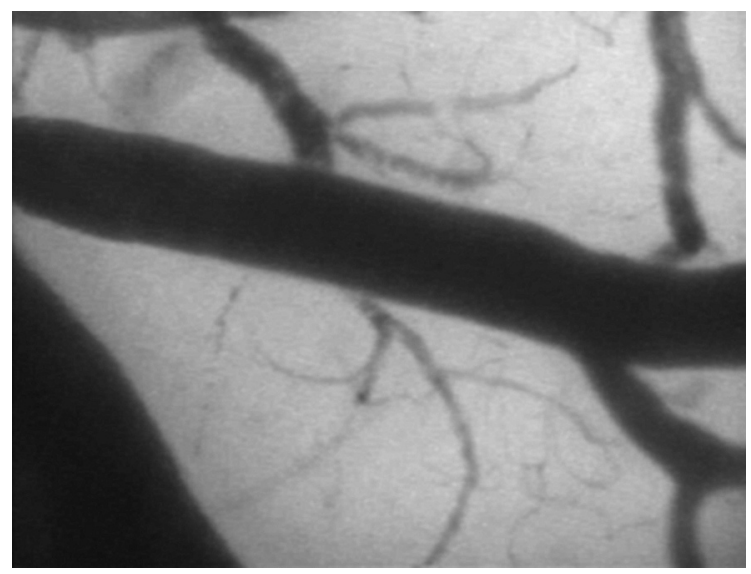

Figura 2. Imagen de la microcirculación de un paciente considerado como control. Se trata de un varón intervenido de forma electiva por un aneurisma cerebral incidental de arteria cerebral media derecha. No presentaba lesiones de ningún tipo en el parénquima. Las imágenes que se muestran se obtuvieron sobre la corteza cerebral de los lóbulos frontal y temporal.

en el caso control los vasos ocupan el 14,17\% del área total de la imagen, mientras que en el paciente con TCE, únicamente el 2,51\%.

\section{DISCUSIÓN}

El sistema SDF consiste en un pequeño microscopio de mano que emite un haz de luz polarizada ortogonal con una longitud de onda de $530 \mathrm{~nm}$, y que permite evitar las interferencias originadas por el reflejo de dicho haz de luz sobre la superficie de los órganos. Este haz de luz es absorbido por la hemoglobina de los eritrocitos, lo que permite su visualización de forma nítida en la microcirculación. La imagen es magnificada entre 5 y 10 veces por una lente y queda grabada en un ordenador portátil para luego analizarla mediante un software específico
TABLA 1. Datos de la microcirculación en un paciente control y un paciente con traumatismo craneal

\begin{tabular}{|l|cc|c|c|}
\hline \multirow{2}{*}{ Grosor } & \multicolumn{2}{|c|}{ Longitud $^{\mathrm{a}}$} & \multicolumn{2}{|c|}{ Área $^{\mathrm{b}}$} \\
\cline { 2 - 5 } & Control & Caso & Control & Caso \\
\hline $0-20 \mu \mathrm{m}$ & $77,87 \%$ & $55,14 \%$ & $6,87 \%$ & $0,73 \%$ \\
$20-40 \mu \mathrm{m}$ & $12,31 \%$ & $41,14 \%$ & $3,11 \%$ & $0,84 \%$ \\
$40-60 \mu \mathrm{m}$ & $6,66 \%$ & $3,54 \%$ & $2,53 \%$ & $0,1 \%$ \\
$60-80 \mu \mathrm{m}$ & $2,71 \%$ & $0 \%$ & $1,37 \%$ & $0 \%$ \\
$80-100 \mu \mathrm{m}$ & $0,4 \%$ & $0 \%$ & $0,29 \%$ & $0 \%$ \\
\hline
\end{tabular}

aPorcentaje de la longitud total de todos los vasos, en función de su grosor, tanto en el control como en el caso clínico.

'Porcentaje del área total de la imagen en función del grosor de los vasos. En este caso la suma de porcentajes no alcanza el 100\%, ya que no toda el área observada está ocupada por vasos sanguíneos.

(MAS $^{\circledR}$, Microvision Medical, Países Bajos) que cuantifica la velocidad de los eritrocitos, el diámetro y la densidad de los vasos en la zona estudiada. Dicha zona de estudio tiene un área de $1 \mathrm{~mm}^{2}$ y se sitúa a una profundidad de 50 a $100 \mu \mathrm{m}$ sobre la superficie cerebral.

El sistema SDF se ha empleado en otras enfermedades como shock séptico ${ }^{13}$, aneurismas ${ }^{14}$ o tumores cerebrales $^{15}$, y ha demostrado ser una metodología factible que permite, de una manera sencilla, la visualización in vivo y de forma no invasiva de la microcirculación en diversos órganos, aunque no ha sido empleada hasta la fecha en pacientes con TCE. La calidad de las imágenes obtenidas mediante esta técnica en estos estudios ha permitido realizar mediciones de diámetros vasculares de hasta $5 \mu \mathrm{m}$. Además, el nuevo software existente en el mercado ha facilitado mucho la cuantificación de estas imágenes.

Obviamente, la principal limitación de este sistema es la necesidad de realizar las grabaciones en quirófano, a diferencia de otras enfermedades, como el shock séptico ${ }^{14}$, en las que las imágenes se obtienen de la mucosa sublingual y, por lo tanto, se pueden estudiar tantas veces como sea necesario. Sin embargo, creemos que este sistema puede contribuir a esclarecer el papel de las alteraciones de la microcirculación cerebral en la hipoxia tisular de los pacientes con un TCEG, al permitirnos visualizar y cuantificar el estado de la microcirculación cerebral en estos enfermos, y compararlo con los correspondientes controles. Puede ayudarnos también a definir de forma más específica estas alteraciones y estudiar a los distintos subgrupos de pacientes traumáticos con lesiones focales y en pacientes que son intervenidos con fines descompresivos en situaciones de swelling cerebral. Otras potenciales aplicaciones en el ámbito de la investigación clínica son la evaluación del efecto de la cirugía o de determinadas maniobras anestésicas intraoperatorias en el estado y la función de la microcirculación cerebral.

Como conclusión, el sistema SDF permite el estudio in vivo y de forma no invasiva de la microcirculación cerebral, además de cuantificar parámetros de densidad vascular y velocidad del flujo sanguíneo. Este sistema puede utilizarse en los pacientes 
con traumatismo craneal para profundizar en el conocimiento de la fisiopatología de la isquemia/hipoxia cerebral postraumática, donde las alteraciones de la microcirculación pueden tener un papel relevante.

\section{BIBLIOGRAFÍA}

1. Marín-Caballos AJ, Murillo-Cabezas F, Domínguez-Roldán JM, Leal-Noval SR, Rincón-Ferrari MD, Muñoz-Sánchez MA. Monitorización de la presión tisular de oxígeno (PtiO2) en la hipoxia cerebral: aproximación diagnóstica y terapéutica. Med Intensiva. 2008;32:81-90.

2. Sahuquillo J, Rodríguez-Baeza A, Pedraza S, et al. Alteraciones de la microcirculación y de la barrera hematoencefálica en los traumatismos craneoencefálicos. Fisiopatología del edema cerebral postraumático.En: NetA, Marruecos-Sant L, editores. Traumatismo craneoencefálico grave. Barcelona: Springer Verlag Ibérica; 1996. p. $14-44$.

3. Zauner A, Bullock R, Kuta AJ, et al. Glutamate release and cerebral blood flow after severe human head injury. Acta Neurochir (Suppl). 1996;67:40-4

4. Graham DI, Adams JH, Doyle D. Ischemic brain damage in fatal non missile head injuries. J Neurol Sci. 1978;39:213-34.

5. Graham DI, Ford I, Adams JH, et al. Ischemic brain damage is still common in fatal non-missile head injury. J Neurol Neurosurg Psychiatry. 1989;52:346-50.
6. Coles JP, Fryer TD, Smielewski P, et al. Incidence and mechanisms of cerebral ischemia in early clinical head injury. J Cereb Blood Flow Metab. 2004;24:202-11.

7. Hutchinson PJ, Gupta AK, Fryer TF. Correlation between cerebral blood flow, substrate delivery, and metabolism in head injury: a combined microdialysis and triple oxygen positron emission tomography study. J Cereb Blood Flow Metab. 2002;22: $735-45$.

8. Menon DK, Coles JP, Gupta AK, et al. Difusion limited oxygen following head injury. Crit Care Med. 2004;32:1384-90.

9. Metz C, Taeger K. Brain damage and cerebral hypoxia. Diagnostics, monitoring, therapy. Anaesthesist. 2000;49:332-9.

10. Siggaard-Andersen O, Fogh-Andersen N, Gothgen IH, et al. Oxygen status of arterial and mixed venous blood. Crit Care Med. 1995;23:1284-93.

11. Rodríguez-Baeza A, Reina F, Sahuquillo J, et al. Relevancia de las alteraciones morfoestructurales detectadas en la microvascularización de la corteza cerebral en pacientes fallecidos por traumatismo craneoencefálico grave. Rev Neurol. 2000;31:911-8.

12. Groner W, Winkelman JW, Harris AG, et al. Orthogonal polarization spectral imaging: a new method for study of the microcirculation. Nat Med. 1999:5:1209-12.

13. De Backer D, Creteur J, Preiser JC, et al. Microvascular blood flow is altered in patients with sepsis. Am J Resp Crit Care Med. 2002;166:98-104.

14. Pennings FA, Bouma GJ, Ince C. Direct observation of the human cerebral microcirculation during aneurysm surgery reveals increased arteriolar contractility. Stroke. 2004;35:1284-8.

15. Mathura KR, Bouma GJ, Ince C. Abnormal microcirculation in brain tumors during surgery. Lancet. 2001;358:1698-9. 